Arteterapia. Papeles de arteterapia y educación para inclusión social ISSN-e 1988-8309

\title{
El arteterapia en el contexto de los Servicios Sociales de Base: el taller de arteterapia como espacio de observación para la intervención primaria
}

\author{
Eva Mayo
}

Recibido: 05 de marzo de 2017 / Aceptado: 29 de julio de 2017

Resumen. Mediante este artículo nos aproximamos a parte de los resultados obtenidos en el estudio cualitativo realizado sobre un taller de arteterapia para la intervención primaria en el ámbito de los servicios sociales de base. Observamos como el arte se configura como una herramienta útil para la intervención social, como un espacio de observación donde detectar problemáticas sociales y dificultades en el desarrollo infantil.

Palabras clave: Arteterapia; Servicios Sociales; Menores; Intervención social.

\section{[en] Art therapy in the context of Basic Social Services: the art therapy workshop as an observation space for primary intervention.}

\begin{abstract}
Through this article, we approach part of the results obtained in the qualitative study carried out on an art therapy workshop for primary intervention in the field of social services. We see how art is configured as a useful tool for social intervention, as an observation space to detect social problems and difficulties in child development.
\end{abstract}

Keywords: Art therapy; Social Services; Children; Social Intervention.

Sumario. 1. Nuevas formas de intervenir: El arte como espacio y herramienta de intervención social. 2. Arteterapia en el ámbito social. 3. Arteterapia en el contexto de los Servicios Sociales municipales. 4. El arteterapia como espacio de observación para la intervención primaria en el contexto de los Servicios Sociales de Base. 5. Conclusiones. 6. Bibliografía.

Cómo citar: Mayo, E. (2017). El arteterapia en el contexto de los Servicios Sociales de Base: el taller de arteterapia como espacio de observación para la intervención primaria, en Arteterapia. Papeles de arteterapia y educación para inclusión social 12, 179-190. 


\section{Nuevas formas de intervenir: El arte como espacio y herramienta de intervención social}

Los cambios socioeconómicos se producen a un ritmo vertiginoso en la sociedad actual. En un espacio global incontrolado, se erosiona el estado de bienestar a través de un mercado que desplaza la acción de los estados, con una reducción del aseguramiento público en beneficio del beneficio, hacia una sociedad del bienestar, un bienestar mercantilizado. Los recursos sociales públicos se merman y precarizan por efecto de la crisis económica, al tiempo, que se aumenta la demanda de ayuda. El individualismo, propio de la sociedad capitalista, determina las relaciones y las caracteriza como precarias y transitorias (Bauman 2005). Se crea así una sociedad donde los lazos solidarios son frágiles, donde la red de apoyo social es débil mientras se desmantelan los servicios públicos. Esta situación es un desafío para el trabajo en lo social.

En una sociedad cambiante, las necesidades y los contextos también cambian. Por ello, los itinerarios de inserción social tradicionales se quedan obsoletos. Los nuevos retos sociales y personales generan nuevas situaciones de exclusión que abordar, nuevas demandas que atender.

El transito a la sociedad postindustrial conlleva transformaciones económicas y sociales, factores determinantes de nuevos riesgos sociales a los que, junto a la globalización y los viejos retos aún desatendidos, el Estado de Bienestar debe enfrenarse (Del Pino y Rubio 2013). Estos nuevos riesgos sociales responden a diversos procesos como son las transformaciones en el mercado de trabajo, el cambio del rol de mujer y su mayor participación en el mundo laboral remunerado, cambios en la configuración de los hogares y las familias, el envejecimiento de la población y las reformas y la privatización de los servicios públicos (Moreno 2010, Taylor-Gooby 2004, Fantova 2007).

Los cambios sociodemográficos y la postindustrialización de las últimas décadas han incidido en el papel de la familia como proveedora de bienestar. Factores como el envejecimiento de la población y la incorporación de la mujer al trabajo remunerado son fenómenos que están modificando la composición de los hogares y el funcionamiento de las redes familiares y comunitarias. El nucleo familiar deja de ser la red de protección y solidaridad fundamental, el soporte social que tradicionalmente había resuelto eficazmente los retos vitales de las personas. El cuidado de los miembros de la familia, antes responsabilidad asumida por las mujeres, se mercantiliza, y la necesidad de atención en el hogar y la demanda de servicios personales generan un mercado privado de servicios sociales.

La globalización reestructura el tejido económico y afianza la deslocalización de los centros de producción, desplazando la mano de obra a países menos desarrollados, e incrementando las exigencias de cualificación del mercado de trabajo. En un bienestar cada vez mas asociado a la participación en el mundo laboral, existen personas incluidas y excluidas socialmente según se encuentran dentro y fuera del mercado de trabajo. De ese modo, las trayectorias laborales cada vez más discontinuas y el desempleo suponen un alto riesgo de exclusión social, que afecta especialmente a personas con baja formación, que optan a puestos en condiciones precarias, bajos salarios y alta inestabilidad. De este modo se enfatiza la dualidad social que señalan Del Pino y Rubio (2013) y que caracteriza la evolución de los modelos de bienestar 
europeos y norteamericanos, donde el estado va delegando su función de proveedores de seguridad y bienestar social.

En sociedades cada vez más duales, con trayectorias vitales más individualizadas y vinculos comunitarios débiles, la ciudadanía se enfrenta a nuevos riesgos sociales como son la fragilidad de la tercera edad, la conciliación de la vida laboral y familiar, la maternidad/paternidad individual, la baja cualificación profesional y una cobertura insuficiente de protección social, pública y privada, con servicios y prestaciones inadecuados, entre otros. (Moreno 2010, Fantova 2007, Del Pino Rubio 2013 )

La crisis económica, afrontada desde la austeridad, ha limitado los recursos disponibles para la atención social lo que, en un contexto tan dinámico, acentúa las tensiones entre las nuevas necesidades sociales y la promoción del bienestar ciudadano, objetivo propio del Trabajo Social. Inevitablemente, ante los nuevos riesgos sociales es necesario reinventarse, reestructurando los programas tradicionales y buscando nuevas áreas y formas de intervención que superen los esquemas tradicionales, de carácter más lineal y asistencial, a través de estrategias que incidan en la prevención. Para Fantova (2016), los nuevos servicios sociales deben atender las necesidades de prevención y de apoyo a la interacción (autonomía funcional e integración relacional) de las personas, desde la comunidad y con servicios de proximidad. Y pone el énfasis en el fortalecimiento de la atención primaria del sistema público de servicios sociales.

$\mathrm{Y}$ ante la necesidad de encontrar nuevas formas de intervención, de acuerdo con Creux (2012), consideramos que el arte es una herramienta que puede proporcionar nuevas vías de trabajo en lo social, un trabajo que desde la interdisciplinariedad de respuesta a las necesidades sociales actuales, en una atención integral que incida en la prevención y la integración social.

Las programas tradicionales se han orientado a resolver las necesidades asociadas a un grupo social minoritario (inmigrantes, mayores, infancia, etc.), que en sí mismo era indicador de riesgo de exclusión. La sola pertenencia a un colectivo considerado de riesgo se ha identificado con una problemática determinada, inevitable, compartida y permanente, que se ha paliado por un servicio social técnico y burocrático, donde poco a nada tiene que aportar la persona atendida.

Las nuevas formas de intervención social se orientan a una atención interdisciplinar e integral de la persona que contribuya al desarrollo del proyecto de vida individual más allá de la demanda inmediata y la necesidad percibida. Superando la perspectiva asistencialista, basada en las deficiencias, se busca avanzar hacia un modelo que promueva cambios duraderos a nivel individual, familia y comunitarios, con la participación de las personas implicadas (Segado 2011). Que la persona reconozca sus propios intereses y necesidades, resuelva sus propios conflictos y adquiera control sobre su propia vida, es algo que una intervención a través del arte puede reforzar. Pero el arteterapia nos permite algo más, porque cuando facilita extraer y resolver conflictos también capacita a la persona y contribuye, por un lado, a detectar situaciones de riesgo social que las demandas verbales no explicitan, y por tanto actuar en el marco de la intervención primaria, $y$, por otro, a prevenir nuevas dificultades sociales al dotar de recursos a las personas para la gestión eficaz de sus propios conflictos.

El aumento en últimos años de las prácticas socioeducativas a través del arte está relacionado con los beneficios propios de la actividad artística ya que el arte, en si mismo, resulta terapéutico y contribuye al desarrollo de la persona. Pero también tie- 
ne efectos en el ámbito social ya que las actividades artísticas incentivan procesos de socialización, integración y cooperación (Molina et al. 2009) y facilitan la relación con el entorno, incluyendo en este personas, los objetos y el espacio. Las actividades artísticas en un sentido amplio (artes plásticas, música, expresión corporal, etc.. ) constituyen una herramienta extraordinaria para el trabajo educativo con diferentes colectivos en situación de vulnerabilidad social ya que promueve la transformación personal y social.

El uso del arte en la intervención social se presenta como un campo dinámico y creciente. Para Serrano (2015), el arte es una herramienta eficaz para el Trabajo Social. Logra que se profundice y se facilite la aplicación de diversos procesos de la intervención social, como la cohesión del grupo, la transformación de los procesos y la participación ciudadana, ya que incide directamente en el desarrollo comunitario, social y personal .

Vemos como, por un lado, que el arte trasciende su valor estético y contribuye al desarrollo de la persona. Por otro, aplicadas en el ámbito social, las Terapias Creativas favorecen la integración social. A través de un proceso de creación, entendido como un proceso vital, la producción artística está encaminada a la elaboración de las situaciones de conflicto y el empoderamiento. De este modo, el arteterapia constituye una aportación de gran interés en la atención multidisciplinar en todos los ámbitos de intervención.

\section{Arteterapia en el ámbito social}

La orientación del arteterapia hacia el ámbito de lo social, surge, en gran medida, de la influencia de Edith Kramer. Ella subrayó la importancia de una intervención grupal, y, en especial, la atención a las interacciones interpersonales en el proceso de intervención terapéutica. El formato de intervención grupal es aplicado en arteterapia cuando se interviene con colectivos sociales concretos, como mujeres, personas sin hogar, víctimas de malos tratos, personas reclusas, etc. Tomando en consideración el grupo cliente, las prácticas de intervención social comparten con el arteterapia un ámbito de actuación común, ya que ambas pueden ir dirigidas a atender a grupos de población con problemáticas sociales diversas.

A finales del siglo XX y principios de este siglo, el arteterapia amplía sus enfoques más allá del psicoanálisis, y comienza a ser sensible a la diversidad y el trasfondo cultural de las personas clientes. Para Suess $(2007,28)$, "el arteterapia no está en un afuera discursivo sino se encuentra atravesada por diversos discursos sociales, reflexiones conceptuales y propuestas prácticas."

Algunas posiciones señalan que, la acción social y el arteterapia, no pueden o no deben estar separadas. Argumentan que no se puede desligar a las personas que se tratan de sus entornos culturales, de donde viven, ya que estos les influyen. Nadie vive en un vacío social, y cada persona constituye una combinación única de genética, crianza familiar, influencias ambientales e historia colectiva (Kaplan 2007, Suess 2007). Para Kaplan (2007) este enfoque implica un compromiso del arteterapia con la acción social. Así mismo, considera que la inclusión de lo social en la disciplina es un deber ético, y propone la figura del arteterapeuta como "activista social" así como un acercamiento del arteterapia a los espacios comunitarios. 
Siguiendo esta línea, y cuestionando la diferenciación entre el tratamiento del cuerpo individual y el social, Hocoy (2007) subraya la necesidad de integración de ambas dimensiones en la práctica arteterapéutica contemporánea. Desde este fundamento, la acción y el compromiso social se incluyen de forma consciente en la práctica del arteterapia, orientando la intervención a través del arte a una transformación tanto individual como social.

López (2010) afirma que las personas participantes en un taller de arteterapia, acarrean consigo distintas necesidades, consecuencia de los cambios sociales producidos en las últimas décadas, que afectan a nivel individual y colectivo, lo que plantea nuevas perspectivas de trabajo a considerar en los procesos de arteterapia. De este modo, las actividades artísticas centradas en la identidad grupal y su contribución en el cambio personal, social y ambiental se transforman en objetivos clave. El compromiso del arteterapia para la acción social y el desarrollo comunitario pasa a ser una cuestión fundamental.

El nexo entre el arteterapia y la intervención social se encuentra, según Hocoy (2007) en la versatilidad de la imagen. Las imágenes tienen la capacidad de traer al plano consciente, no solo la realidad individual, sino también la realidad colectiva. Siguiendo a Cassirer (1955 citado por Hocoy 2007), podemos afirmar que la imagen tiene el potencial de mediar y manifestar la conciencia, individual y colectiva, a través de las formas simbólicas que representa. Su capacidad de transformación parte de la concienciación de la existencia de la problemática social, primer paso para el cambio.

El arte como herramienta de intervención social puede ser útil en diferentes circunstancias. El arteterapia, por su parte, también resulta ser de gran utilidad para tratar problemáticas con distintos colectivos en situación de dificultad: niños y adolescentes en casas de acogida, personas inmigrantes, personas mayores, colectivos de personas reclusas, personas victimas de maltrato, mujeres, personas sin hogar y en general, personas o colectivos en situación de riesgo o exclusión social como acreditan los programas de atención social que se implementan de forma habitual en otros países como Reino Unido.

Ascensión Moreno (2006) destaca que las artes, en general, son una herramienta muy valiosa y utilizada en la intervención social, y que el arteterapia, en particular, puede contribuir a mejorar la tarea educativa desarrollada en los talleres artísticos.

\section{Arteterapia en los Servicios Sociales municipales}

El uso del arte para la atención social se ha relacionado tradicionalmente con la animación sociocultural, la educación de adultos y la educación especializada. Ludotecas para menores o programas en centros residenciales que incluyen actividades artísticas, muchas veces a modo de taller de manualidades, son aplicaciones muy extendidas. El Observatorio del Tercer Sector de Bizkaia recoge en el informe "Arte para la inclusión y la transformación social" (2012) numerosas y variadas experiencias de intervención social con apoyo de herramientas artísticas. Tomando como referencia este trabajo, nos proponemos ampliar el conocimiento sobre el uso del arte en la intervención social con una aproximación al ámbito de Servicios Sociales en Bizkaia, de titularidad pública. En relación a las aplicaciones del arte presentadas 
en el estudio referido, y trascendiendo el taller artístico y el enfoque del arte como mediador, consideramos de interés profundizar en la aplicación social del arteterapia, disciplina de incorporación reciente al contexto español, y más novedosa aún en el entorno vasco.

Desde el convencimiento de que la interrelación de diferentes disciplinas enriquece la intervención social, y poniendo en relación el arteterapia y los Servicios Sociales, nos planteamos la siguiente pregunta: ¿Contribuye la utilización del arteterapia a la mejora en la intervención socioeducativa en el ámbito de los Servicios Sociales de Base?.

Para dar respuesta a esta cuestión, tomamos como objeto de investigación el arteterapia en el contexto Servicios Sociales de Base, en el estudio cualitativo de una experiencia que consideramos relevante, tanto por la aplicación del arteterapia como por lo novedoso de esta práctica en ámbito de los servicios sociales municipales en Bizkaia. Con este trabajo nos propusimos poner en valor el uso del arteterapia como recurso de intervención en el ámbito de la atención social, para lo que estudiamos la experiencia que se lleva acabo en el municipio de Leioa (Bizkaia), un taller de arteterapia en el marco de los programas de intervención primaria del Equipo de Intervención Socioeducativa (EISE) en el Servicio de Infancia, Juventud y Familia, una actividad que responde a la búsqueda de nuevos espacios y formas de intervención. En este acercamiento, no nos hemos ceñido únicamente al análisis del impacto en las personas usuarias de la actividad, sino que hemos abordado también aspectos relacionados con la práctica profesional y las características de intervención social con el propósito de dar respuesta a la cuestión inicial. En relación a la intervención social, la hipótesis de trabajo fue "El espacio del arteterapia es un espacio de observación que contribuye a detectar situaciones de riesgo social."

El estudio de esta experiencia, el arteterapia en el contexto de los Servicios Sociales de Base a través del taller de arteterapia, no se plantea la representatividad, y el uso de la metodología cualitativa ha permitido profundizar en las situaciones concretas y analizar los discursos que generan en el contexto del caso, estudiándolo desde la proximidad.

Dentro de la metodología cualitativa, para obtener información se han utilizado en concreto el análisis documental y las entrevistas a informantes clave. También se presentan clasificaciones, atributos y propiedades cuantificables, siento utilizada la metodología cuantitativa para definir los perfiles de participación en las sesiones de arteterapia en la descripción del taller.

Las entrevistas se han realizado a informantes clave "observadores privilegiados, personas conocedoras expertas del fenómeno, con una visión directa y profunda del mismo que los sitúa en una posición de observación privilegiada." (Corbetta 2010). Para la selección de las personas a entrevistar se ha utilizado la Técnica de bola de nieve. Esto es, a partir de una primera persona informante, que coordina el EISE de los Servicios Sociales, se han encontrado nuevas personas informantes, que participan en el desarrollo la actividad del taller de arteterapia con diferente responsabilidad y función.

La muestra no probabilística de las personas entrevistadas está compuesta por cinco personas conocedoras directas de la experiencia, y una persona conocedora del tema pero no implicada directamente. Estas personas son profesionales de los ámbitos social, escolar, cultural y terapéutico que participan coordinadamente en el desarrollo de la actividad: educadoras sociales, arteterapeuta, consultora escolar 
y coordinadora del museo. Todas las entrevistas realizadas han sido individuales, presenciales y telefónicas, semiestructuradas y focalizadas en el tema de estudio, el taller de arteterapia y la implicación en el mismo de la persona entrevistada. Partiendo de un guión flexible, se han abordado contenidos relacionados con los cambios observados en los niños y las niñas participantes, la detección de problemáticas o necesidades personales y sociales, las intervenciones realizadas y la naturaleza de la relación con los/as menores y el taller.

Haurtxoko es el programa marco del taller de arteterapia estudiado. Se trata de un programa dirigido a menores, de entre 4 y 13 años, residentes en el municipio, con indicadores de vulnerabilidad a nivel personal, familiar o social. Desde el servicio intervención primaria, este programa pretende detectar las situaciones de riesgo que dificulten el adecuado desarrollo personal y social de los niños y las niñas, siendo por tanto de carácter preventivo. Así mismo, esta intervención también tiene entre sus objetivos favorecer el desarrollo integral y armónico en la infancia. Para ello, en el contexto de las actividades grupales, se realizan propuestas y dinámicas que permitan trabajar aspectos relacionales, habilidades sociales, gestión de conflictos y la expresión y elaboración emocional. Entre las distintas actividades y metodologías a las que se recurre, para favorecer la participación y alcanzar los objetivos propuestos, se encuentra el Taller de Arteterapia que se implementa a través del Museo de Bellas Artes de Bilbao.

En el contexto del EISE, el objetivo del Taller de Arteterapia es acompañar a los niños y las niñas a vivir nuevas experiencias enriquecedoras que les permitieran dar salida a su mundo emocional. Con ello, se proporcionan nuevas formas de expresión y elaboración emocional que contribuyen a su desarrollo integral, y se constituye un espacio de observación.

Los niños y las niñas que participan en el taller, en general, presentan unas características sociales y personales similares. Estos rasgos se detectan por medio de la observación de los indicadores de orientación del profesorado en los centros educativos locales para la derivación a programas de prevención primaria en el EISE, que son tenidos en cuenta para valorar la propuesta de participación en la actividad de arteterapia.

La metodología es arteterapéutica, desde un enfoque psicoanalítico. Se trata de una práctica psicoterapéutica basada en la expresión, la comunicación y la reflexión sobre los estados emocionales a través de los materiales artísticos y procesos creativos. Partiendo del concepto de espacio potencial de Winnicott, se realiza un trabajo en el espacio intermedio, entre el mundo interno y externo, un espacio en el que se ensaya y se experimenta a través del juego y la creación, con una metodología no directiva. Se realiza una indagación personal "intensa y transformadora" (Lund, 2014) ya que, con la creatividad, la persona es capaz de desarrollar estrategias flexibles y ajustadas a la realidad, y adaptarse aunque esta cambie .

En el desarrollo del taller se encuentran una figura educadora del Equipo de Intervención Socioeducativa de los Servicios Sociales y otra persona arteterapeuta. La función de la arteterapeuta es dirigir el taller. La función de la persona educadora del EISE es el acompañamiento durante todo el proceso, incluido el tiempo de desplazamiento hasta y desde el museo, un tiempo activo y de relación. Se establece un limite máximo de 10 niños y niñas por sesión. En el momento de la recogida de información para el estudio el grupo esta formado por 7 menores.

La actividad se desarrolla dentro de dos espacios diferenciados dentro del Museo: el espacio de exhibición y el espacio de taller. En relación a la temporalización, se 
trata de sesiones de 2 horas de duración, donde se diferencian 3 momentos: uno inicial de interacción con una obra de arte, un segundo momento de creación artística y finalmente el tiempo para el dialogo. El momento inicial se desarrolla en el espacio de exhibición del museo. Previamente, la arteterapeuta ha seleccionado una obra de arte de la colección del museo sobre la que se va a trabajar con el grupo, una pieza artística que observa y con la que se interacciona a través de un dialogo dirigido por la propia arteterapeuta. Se orienta la conversación para ponerla en relación con la vida diaria de los niños y las niñas. Esta práctica suele durar unos 20 o 30 minutos. El momento para la creación se desarrolla en el espacio del taller, donde existen unas normas de funcionamiento que son conocidas y deben ser respetadas por quienes acuden. Estas normas se clarifican al inicio de la sesión. Es un espacio de creación, donde tienen a su disposición materiales (plásticos, artísticos, de reciclaje, etc.) para realizar sus obras, que habitualmente responden a un trabajo individual que permita la expresión personal. Lo importante es el proceso, y por tanto no hay un criterio estético en relación al trabajo que se realiza. Disponen de unos 60 minutos para la elaboración de su propuesta plástica. El tercer momento, que también se desarrolla en el espacio del taller, se caracteriza por un dialogo dirigido por la arteterapeuta. Es una puesta en común del trabajo realizado desde lo simbólico, desde la libertad, la seguridad y la no obligación. Se acepta el silencio como forma de participación. Este proceso suele requerir unos 30 minutos.

\section{El arteterapia como espacio de observación para la intervención primaria en el contexto de los Servicios Sociales de Base}

La actividad de arteterapia se realiza en el marco de la intervención primaria de los Servicios Sociales de Base que asume el Equipo de Intervención Socioeducativa. Las intervenciones preventivas grupales, son espacios de "ver que les está pasando(a los/as menores), y de expresión y elaboración de esa vivencia emocional que puedan estar teniendo." (persona entrevistada 2). Constituyen por tanto un espacio de observación. Del mismo modo, el taller de arteterapia permite, desde la expresión libre y la elaboración emocional en un entorno seguro, poner de relieve aspectos de la vida de los/as menores que pueden constituir un riesgo para su desarrollo personal y social. Con su expresión artística, los niños y niñas participantes de un modo simbólico exteriorizan sus vivencias y sus temores, pudiendo encontrar una vía para compartir sus anhelos en relación a su vida, personal, social y familiar. Esta detección precoz de una situación de necesidad no verbalizada, de una carencia no satisfecha, permite una intervención temprana, propia de la acción preventiva, que evite efectos no deseados atendiendo prematuramente. Resulta especialmente interesante en aquellos casos en los que por sus circunstancia no son capaces o bloquean la expresión de conflictos, que cuando hablamos de menores, una parentalidad de riesgo puede no ser percibida o verbalizada por el niño o la niña ya que genera un conflicto de fidelidad a la estructura familiar.

Destacamos como, desde el ámbito socioeducativo la persona entrevistada 2 afirma que se han detectado nuevas problemáticas o necesidades durante el desarrollo de la intervención arteterapéutica. La persona educadora social del EISE, desde su función de acompañante, puede obtener información valiosa sobre los niños y las niñas, 
ya que como afirma la persona entrevistada 4, "hay cosas que surgen en el desarrollo de las sesiones". En ocasiones, un niño o una niña que acude al taller dispone de una valoración psicológica previa, pero también se realizan aportaciones y devoluciones fruto de la intervención arteterapéutica. Con ello no se pretende la confirmación de un diagnostico previo, ni realizar un tratamiento psicológico, sino canalizar la necesidad hacia la intervención más adecuada que permita mejorar la vida de la niña o del niño. El arteterapia permite la escucha y da visibilidad a las vivencias emocionales infantiles, y dota de recursos al grupo para gestionar sus conflictos reforzando así sus capacidades.

Desde el ámbito de la orientación educativa, la persona entrevistada 3 señala, en referencia al alumnado, "se cierran" cuando verbaliza su situación personal. El control del lenguaje verbal es algo que dominan, saben callar y esconder, no expresan lo que les pasa con palabras, por miedo, vergüenza o simplemente no lo saben. Pero gracias a que "las terapias creativas abren ranuras" (persona entrevistada 3 ), han salido a la luz nuevas problemáticas del alumnado fruto del trabajo de gestión emocional realizado en las sesiones de arteterapia que contribuye a mejorar la comunicación verbal y facilita al alumnado compartir sus inquietudes. Hablamos de situaciones de dificultad relacionadas no solo con el contexto escolar, sino también vivencias relacionadas con las dinámicas familiares que estaban afectando al desarrollo personal del niño o la niña, que se atendieron con la derivación a los servicios sociales pertinentes. Para la persona entrevistada 3, a través del arteterapia lo que se hace "es un poco poner voz", y que "salen cosas y hay que poner las piezas en su lugar".

Cuando hablamos detección de dificultades en los niños y las niñas que puedan afectar a su desarrollo no hablamos de preguntar al niño o la niña, que es lo que le pasa y recoger la respuesta. La intervención primaria que se realiza desde el espacio de observación que constituye el taller de arteterapia, no responde a demandas explicitas. Desde el lenguaje simbólico, a través del arte, se genera un dialogo con el interior que conecta con el exterior que permite aflorar los conflictos personales. En este sentido, la persona entrevistada 2 afirma, en relación a la expresión artística y el arteterapia, que "no es solo un lenguaje diferente, también es una escucha diferente". Como sostenía Kramer (1982), el lenguaje artístico permite a las personas comenzar a manifestar las cosas que les preocupan, que les atormentan, entendiendo que la expresión no es una traducción de la vida interior sino una representación. $\mathrm{La}$ imagen no es literal ni tiene valor estético.

\section{Conclusiones}

Del análisis de los datos registrados durante las entrevistas a informantes clave y de fuentes documentales manejadas, se confirma la hipótesis de partida: El espacio del arteterapia es un espacio de observación que contribuye a detectar situaciones de riesgo social. Desde todos los ámbitos implicados en la implentación del taller, social, escolar, terapéutico y cultural, se evidencia que la información que aportan los niños y las niñas de sí y sus vivencias durante las sesiones permite detectar indicadores de riesgo en su desarrollo social y personal, que en referencia a casos concretos, se han derivado para intervenciones secundarias. 
Las artes favorecen la expresión de las inquietudes, necesidades y la proyección de los conflictos internos que no pueden ser expresados con palabras, y el espacio de arteterapia es un entorno facilitador. Es el sacar a través del arte lo que permite tener algo que observar, y así detectar la necesidad del niño o la niña. Por ello, el arteterapia constituye un recurso útil para el enfoque preventivo de la intervención primaria en el contexto de los Servicios sociales.

Observamos que los trabajos artísticos se pueden utilizar como instrumentos de diagnostico como señalaban Gonzalez Barroso y Garcia Moro (2005). Los conflictos internos que se proyectan a través del arte proporcionan una información valiosa que facilita la evaluación de diagnostico para la intervención social, y el taller es el espacio que permite observar y recoger datos de esas proyecciones. Encontramos como, los dibujos y narraciones que realizan los niños y las niñas han servido para detectar situaciones de riesgo social. Y a partir de esa detección, actuar y alcanzar así los objetivos propios de la intervención social de carácter preventivo en los Servicio Sociales: evitar el desarrollo de un problema o necesidad dotando de recursos a la persona para afrontarlo.

En el estudio de la actividad de arteterapia desarrollada en el marco de la intervención primaria, nos presenta el taller como un espacio seguro para la expresión de quienes participan y una herramienta útil para la atención social preventiva. En el taller la comunicación de las necesidades trasciende la mera expresión verbal y utiliza el lenguaje plástico de forma libre. Su carácter lúdico disuelve las resistencias de los niño y las niñas, que se dejan llevar, en un entorno cómodo y accesible donde comparten sus vivencias, lo que facilita detectar de forma temprana situaciones de dificultad social y personal que afecten su desarrollo. Cuando no son conscientes de sus propias problemáticas, el proceso artístico exterioriza y pone sobre la mesa aspectos sobre la vida de un niño o una niña que con las palabras no pueden o no saben compartir. El arteterapia facilita un espacio donde la elaboración emocional a través del objeto artístico, en su dimensión simbólica y no estética, permite no solo compartir de sus preocupaciones, miedos y deseos, sino que proporciona aprendizajes valiosos para los niños y las niñas. Es destacable como adquieren competencias para el manejo de las emociones, lo que les proporciona habilidades para la gestión de de conflictos. Estos y otros aprendizajes suponen un fortalecimiento de los recursos personales que contribuyen a mejorar la competencia social y mejoran su autoestima, posibilitando un desarrollo más equilibrado y integración social mas adecuada.

Además de las observaciones anteriores referidas a la hipótesis de estudio, el taller de arteterapia presenta un encaje adecuado en el contexto de los Servicios Sociales de Base. Esta actividad, enmarcada en el programa Haurtxoko, contribuye a alcanzar los objetivos de la intervención con menores que se proponen desde la prevención primaria en la atención social de base: detectar las situaciones de riesgo y favorecer el desarrollo armónico e integral de los niños y las niñas. Desde este enfoque integral, es importante subrayar la interdisciplinariedad de la intervención, en la que participan agentes del ámbito escolar, terapéutico, cultural y de los propios servicios sociales. Esto implica un compartir objetivos propios de las diferentes disciplinas. Así, los objetivos socioeducativos apuntados confluyen con los objetivos propios del arteterapia que, como señala Kaplan (2007), son contribuir al cambio personal y social. Y también asumen los objetivos escolares para el desarrollo del alumnado emocionalmente equilibrado que se explicitan en los proyectos educativos de los colegios. 
Además de facilitar los objetivos propios de los servicios sociales, esta forma de intervención, interdisciplinar y con un enfoque integral y no meramente asistencial se encuentra en la linea de las propuestas e inquietudes de la práctica de la atención social. Si bien atiende al colectivo infantil, y toma como referencia las características sociales generales de los niños y niñas que acuden al taller como el género o la etnicidad, el arteterapia se centra en la persona y en su actuación toma en consideración la trayectoria y el proyecto individual, del que se realiza un seguimiento en común con las profesionales que se implican en el proceso de intervención. Coincidimos con Moreno (2006) cuando apunta que el arteterapia puede aportar elementos metodológicos y conceptuales que mejoren las prácticas educativas que se realizan desde los servicios y centros de la red social. Y que con la interrelación las disciplinas se enriquecen mutuamente. En este caso, el arteterapia se manifiesta como una herramienta que contribuye positivamente a la intervención primaria en el contexto de los Servicios Sociales de Base. Con el producto artístico y la elaboración simbólica, las personas adquieren un control sobre si mismas con el que se perciben competentes para tomar decisiones y resolver conflictos, lo que beneficia la implicación en su propio proyecto vital y se consolida como un recurso para la prevención de la exclusión social.

Este, solo ha sido el acercamiento a una posibilidad que el arteterapia proporciona como herramienta para la intervención social en el contexto de los Servicios Sociales de Base. En la búsqueda de nuevas formas de intervenir, el arteterapia se revela como una oportunidad para la innovación en la práctica del trabajo social. Permite cambios duraderos de las situaciones, conlleva aprendizajes que dotan de recursos propios a las personas y aborda la vulnerabilidad social más allá de la necesidad inmediata, superando el enfoque de intervención tradicional que resulta ineficaz en un contexto social tan dinámico como el actual. Pero, como ya señalaba Creux (2012), quienes trabajan en la intervención social se encuentran con la demanda de actuar según los procedimientos. Y esta necesidad primera de cumplir con los servicios concretos establecidos en el sistema de servicios sociales, y lo limitado de los recursos que manejan los organismos municipales, no facilita la incorporación de otras posibilidades de hacer como lo es la utilización del arteterapia en el marco de los programas de prevención primaria.

Pero aunque la innovación en el ámbito social se encuentra con limitaciones en la práctica, en la aplicación del arteterapia para la intervención primaria y secundaria, se abren nuevas posibilidades de indagación que pueden contribuir a completar un corpus teórico propio. En relación a la metodología, nos preguntamos, ¿puede definirse una metodología arteterapéutica especifica para el trabajo en el ámbito de los Servicios Sociales Municipales? Con respecto al contexto de los Servicios Sociales, ¿tiene cabida el arteterapia del mismo modo que se hace en otros países del entorno, como un servicio público más? ¿Tendría encaje en el conjunto de los Servicios Sociales de Base?. El arteterapia como disciplina en construcción, admite aún más interrogantes, esta preguntas son una propuesta más para continuar esa construcción. 


\section{Bibliografía}

Carnacea, A. y Lozano, A. Coord. (2011). Arte, intervención y acción social. La creatividad transformadora. Madrid: Editorial Grupo 5.

Castillo, R, Sostegno, R y López-Arostegi, R. 2012. Arte para la inclusión y la transformación social. Bilbao: Observatorio del Tercer Sector de Bizkaia.

Creux, G. (2012). "Art and social work. Towards a sociological analysis ” Bordeaux. Bordeaux.

Dalley, T. (1987). El arte como terapia. Barcelona: Editorial Herder.

Del Pino, E y Rubio, MJ. Ed.(2013). Los estados de bienestar en la encrucijada. Politicas sociales en perspectiva comparada. Madrid: Tecnos.

Dominguez, P.M. Coord. (2005). Arteterapia. Principios y ámbitos de aplicación. Sevilla: Junta de Andalucia.

Fantova, F. (2016). "Nuevos enfoques para los servicios sociales ante la nueva realidad social”. Revista Española del Tercer Sector 33:115-139. Madrid: Luis Vives.

Kaplan, F. (2007). Art Therapy and Social Action: Treating the World's Wounds. Londres: Jessica Kingsley Publishers.

López, M. Coord. (2006). Creación y posibilidad. Aplicaciones del arte en la integración social. Madrid: Editorial Fundamentos.

López, M.D. (2009). La intervención arteterapéutica y su metodología en el contexto profesional español. Murcia:Universidad de Murcia.

Moreno, A. (2003). Aportaciones del arteterapia a la educación social en medio abierto. Barcelona: Universidad de Barcelona.

Subirats, J. Dir. (2007). Los Servicios Sociales de Atención Primaria ante el cambio social. Madrid: Ministerio de Trabajo y Asuntos Sociales.

Suess, A. (2007). “Arte, terapia y transformación social en la intersección entre postestructualismo y teoría crítica". Arteterapia - Papeles de arteterapia y educación artística para la inclusión social 27 Vol. 2: 27-37. Madrid; UCM. 